\title{
Primary Peritoneal Malignant Mixed Müllerian Tumor in a Young Woman: Achieving the Best Clinical Benefit
}

\author{
E. Uña ${ }^{a} \quad$ M. García-Tejeiro ${ }^{b} \quad$ M. Álvarez ${ }^{b}$

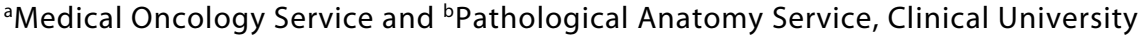 \\ Hospital of Valladolid, Valladolid, Spain
}

\section{Key Words}

Peritoneal tumors - Malignant mixed müllerian tumors · Extragenital tumors ·

Carcinosarcomas

\begin{abstract}
Extragenital malignant mixed mesodermal müllerian tumors (MT) are rare neoplasms with poor prognosis. Most of them affect women older than 60 years. We present here a case with primary peritoneal malignant mixed müllerian tumor occurring in a young woman who underwent previous hysterectomy and double oophorectomy secondary to a benign disease. We report on the clinical, pathological, and immunohistochemical features of this lesion, which was composed of a poorly differentiated epithelial component and multiple areas of chondromatous differentiation. Along with a brief review of previously reported literature about genital and extragenital MT, some concepts relevant to this case are discussed.
\end{abstract}

\section{Introduction}

Malignant mixed müllerian tumors (MT) are a rare mixed mesenchymal and epithelial group of neoplasms [1]. Although these tumors occur most commonly in the endometrium and less frequently in other parts of the female genital tract, such as the cervix, ovaries, fallopian tubes and vagina, extragenital primary peritoneal MT that are extremely rare have been described, and only few cases have been reported in the literature $[1,2]$. These tumors occur commonly in elderly postmenopausal women $[1,2]$ and it has been hypothesized that they arise in the secondary müllerian system consisting of the abdominal and pelvic peritoneum and its underlying mesenchymal tissue. They are considered neoplasms with a highly aggressive course and very poor prognosis [2]. 


\begin{tabular}{|c|c|c|c|}
\hline $\begin{array}{l}\text { Cose Reports in } \\
\text { tincisy }\end{array}$ & $\begin{array}{l}\text { Case Rep Oncol 2009;2:162-167 } \\
\text { D0I: } 10.1159 / 000235912\end{array}$ & Published online: September 8, 2009 & $\begin{array}{l}\text { (c) } 2009 \text { S. Karger AG, Basel } \\
\text { ISSN } 1662-6575 \\
\text { www.karger.com/cro }\end{array}$ \\
\hline
\end{tabular}

\section{Case Report}

A 46-year-old woman was admitted to the hospital complaining of diffuse abdominal pain and swelling, nausea and decrease of appetite. Abdominal examination revealed multiple abdominal masses and ascites. She underwent a hysterectomy and oophorectomy 6 years ago, secondary to a benign disease; other than this, there was no remarkable gynecological history.

Abdominal ultrasound and computed tomography revealed multiple peritoneal masses with a diameter of 4-10 cm. A vaginal examination was unremarkable. She underwent drainage of the ascites and subsequent cytological study revealed adenocarcinoma. Inmunohistochemistry did not help in the diagnostic process. An upper digestive endoscopy and colonoscopy were normal. Chest X-ray was normal, too.

Laparotomy achieved total resection of a bleeding abdominal tumor, measuring $10 \mathrm{~cm}$ at its maximal diameter, attached to the retroperitoneal area, and most of the peritoneal masses leaving approximately $40 \%$ of the visible disease. The postoperative course was difficult. The patient had an Eastern Cooperative Oncology Group (ECOG) Performance Status of 3, with diffuse edemas, loss of appetite, fatigue and dispnea at moderate physical efforts. After a short course of high doses of steroids such as anabolic drugs, intravenous albumin, diuretics, morphine and blood transfusions, the patient achieved an ECOG of 1-2 after two weeks and she could leave the hospital.

Pathologic findings revealed mesenteric tissue diffusely invaded and distorted by a soft tumor. This tumor was predominantly solid with areas of hemorrhage. Light microscopy showed a tumor with a biphasic pattern. The larger part of the tumor consisted of cords and nests of poorly differentiated intermediate-sized cells. Several nuclei displayed a dispersed chromatin pattern. There was a high mitotic rate. The tumor was composed of a mixture of carcinomatous and sarcomatous components (fig. 1). The carcinoma component was formed by indifferentiated diffuse cells and glandular structures composed of larger cells focally resembling adenocarcinoma. The stromal component consisted of chondromatous areas (fig. 2), spindle cells with pleomorphic nuclei, mostly with a haphazardous architecture, and pleomorphic giant cells in some areas.

The immunohistochemical findings showed expression of cytokeratins (fig. 3 ) and smooth muscle actin ( $\underline{\text { fig. }}$ ) in the epithelial and mesenchymal components, respectively, and the absence of desmin and estrogen and progesterone receptors.

Postoperatively, the patient was given chemotherapy with a combination of paclitaxel and carboplatin in a weekly schedule because of the regular general condition. The patient experienced clinical benefit and marked decrease of serum levels of CA 125 after two cycles. At that time, we modified the schedule to every 3 weeks. Now, she is well and she continues receiving chemotherapy 6 months after diagnosis. Computed tomography after 4 cycles revealed partial response.

\section{Discussion}

MT are uncommon neoplasms characterized by their biphasic pattern containing both malignant epithelial and stromal components [1]. These tumors most commonly arise in the uterus and other parts of the female genital tract, but the occurrence of MT in an extragenital location, although it exists, is very rare. There are different sites for extragenital MT and the most frequent location appears to be the pelvic peritoneum. Other sites are serosal surface of the colon, retroperitoneum, antero-lateral abdominal peritoneum and the mesentery [2].

Peritoneal surfaces are a host to a range of benign and malignant diseases commonly encountered in the müllerian duct system of the female genital tract [3]. Primary peritoneal MT have been thought to arise either from foci of endometriosis, from müllerian duct remnants or directly from the mesothelium and submesothelial mesenchyme through a process of metaplasia. This müllerian potential of the peritoneal serosal and adjacent mesenchyme makes it evident that they share mesodermal ancestry with the primary müllerian system, which derives from invaginated coelomic epithelium. 
Lauchlan called the areas 'secondary müllerian system' which are present in the peritoneum and not restricted to females $[3,4]$.

In reviews of cases of peritoneal MT, this disease was found to be associated with synchronous gynecologic tumors of müllerian duct origin (i.e., ovarian tumors, primary serous carcinoma of the peritoneum, fallopian tube cancer, endometrial cancer and cervix adenocarcinoma) or colonic carcinomas and serous carcinoma of the peritoneum [5-8]. Although this coexistence may be incidental, it may also imply a possible linkage between these tumors. Sometimes, these tumors may appear along the clinical evolution of the first neoplasm (metachronic) [5].

In most cases, MT including genital and extragenital locations are described in postmenopausal women older than 60 years who present risk factors such as obesity, nulliparity, exogenous estrogen or long-term tamoxifen use [1].

Here, we report a case of a young woman with MT stage IIIC of abdominal peritoneum origin without other associated tumors. The patient was operated for hysterectomy and double oophorectomy 6 years before the diagnosis because of a benign disease; she has no other relevant gynecological history and did not present any of the known risk factors for this neoplasm.

The pathologic stage at diagnosis seems to be the most reliable predictor of prognosis in MT of uterine origin [3]. Although usually the prognosis of MT is poor, it seems to improve with combinations of treatments. Surgical excision is the most effective treatment and radiotherapy and various combinations of chemotherapy have achieved inconsistent results [9]. However, Wong et al. observed that patients with MT of the uterus who received sequential adjuvant therapy, using cisplatin and ifosfamide chemotherapy and radiotherapy, had an improved survival rate [10].

In our case, the patient was subjected to suboptimal cytoreductive surgery but achieved a clinical benefit. After that, the patient at first begun a combination of chemotherapy consisting of paclitaxel and carboplatin in a weekly schedule because of the regular general condition. She tolerated the therapy well and presented with a marked decrease in serum levels of CA 125. This is the first case reported according to our knowledge in which the schedule of chemotherapy is performed weekly and with this combination of drugs. It is relevant to bear these results in mind because this schedule might be an alternative to combination of cisplatin and ifosfamide, which are more toxic drugs.

In summary, we report on a primary peritoneum neoplasm, exhibiting both carcinomatous and sarcomatous features, in a young woman without known risk factors who experienced a marked clinical benefit after cytoreductive surgery with a maximal effort and chemotherapy with a well-tolerated schedule. 


\begin{tabular}{|c|c|c|c|}
\hline $\begin{array}{r}\text { Case Reports in } \\
\text { Encisily }\end{array}$ & $\begin{array}{l}\text { Case Rep Oncol 2009;2:162-167 } \\
\text { D0I: } 10.1159 / 000235912\end{array}$ & Published online: September 8, 2009 & \begin{tabular}{|l} 
@) 2009 S. Karger AG, Basel \\
ISSN 1662-6575 \\
www.karger.com/cro
\end{tabular} \\
\hline
\end{tabular}

Fig. 1. Microphotograph-mixture of carcinomatous and chondromatous sacromatous components.

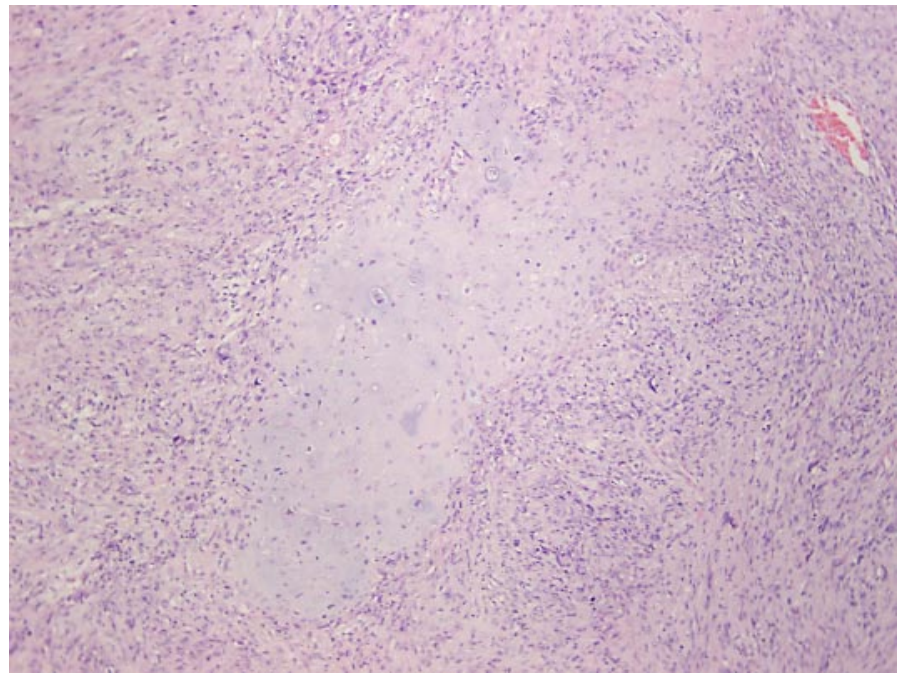

Fig. 2. Microphotograph showing chondromatous sacromatous component.

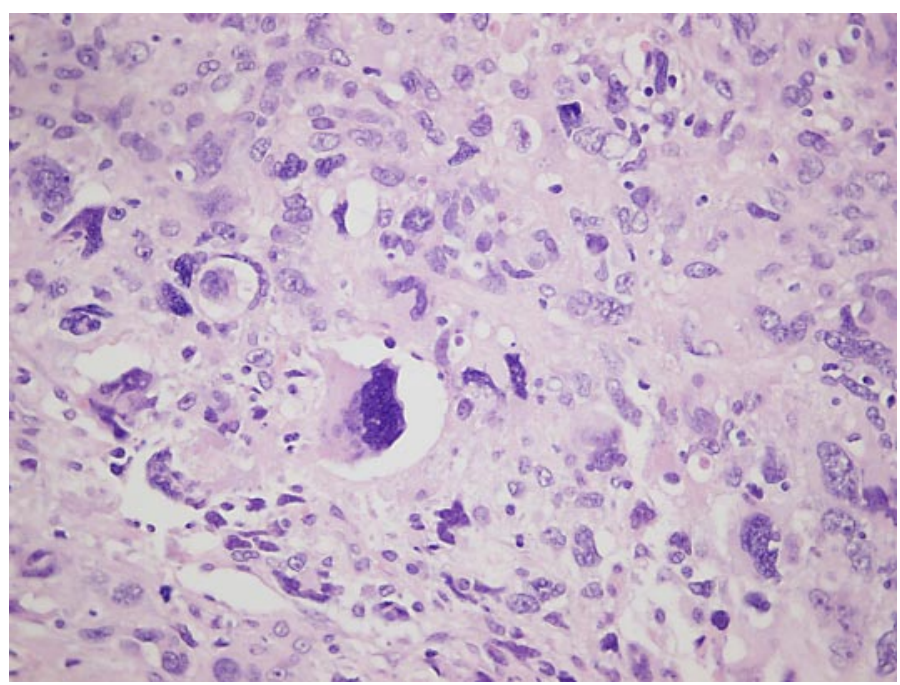


Fig. 3. Immunohistochemical studies showing immunoreactivity for cytokeratin AE1 in epithelial component whereas the coexisting sacromatous component was negative.

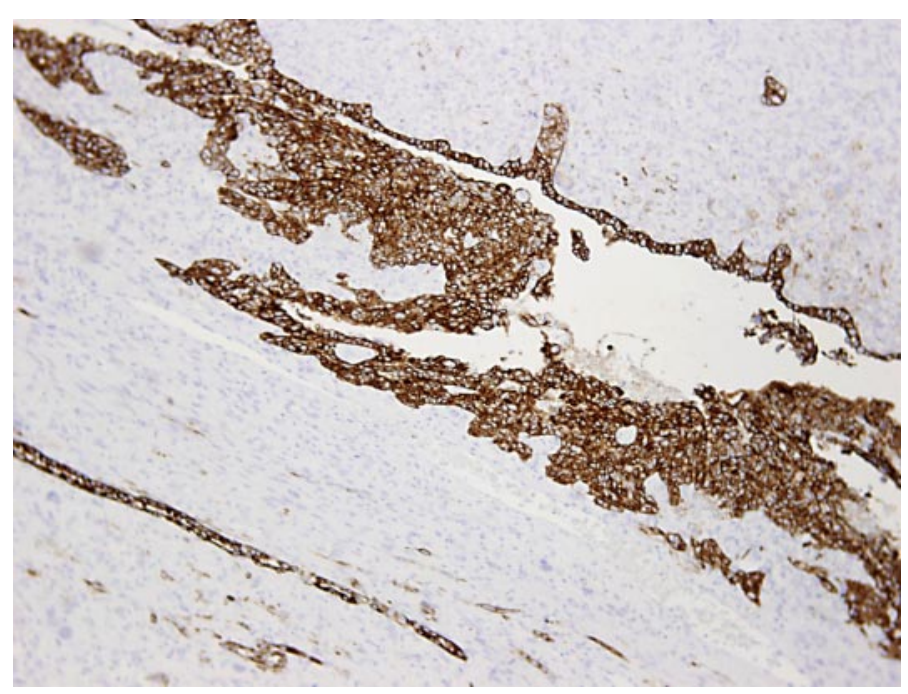

Fig. 4. Immunohistochemical studies demonstrate immunoreactivity for smooth muscle actin (SMA).

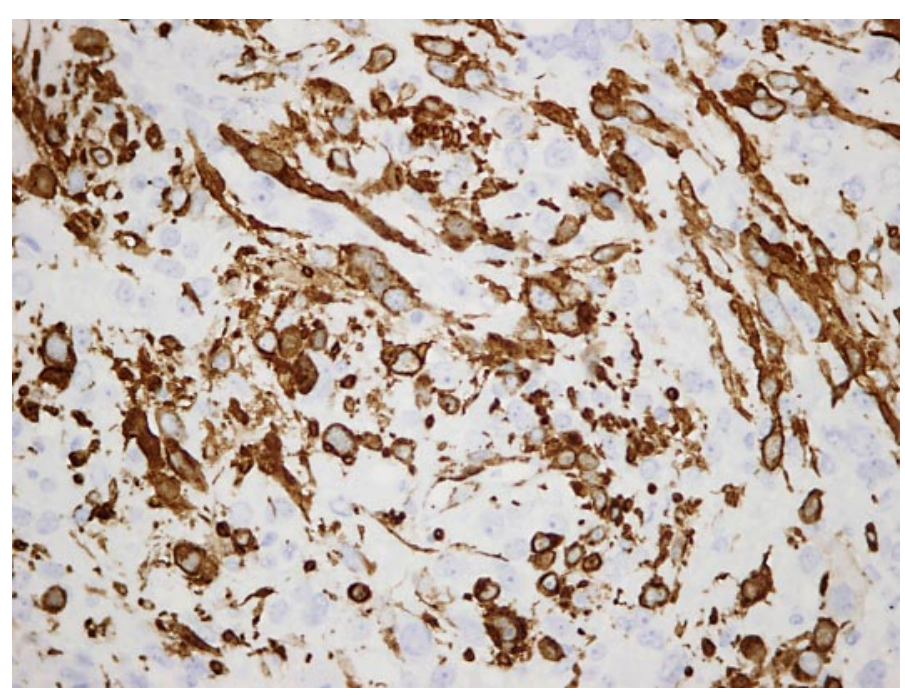




\section{References}

1 Shen DH, Khoo US, Xue WC, et al: Primary peritoneal malignant mixed müllerian tumors. A clinicopathologic, immunohistochemical and genetic study. Cancer 2001;91:1052-1060.

2 Naniwadekar MR, Desai SR, Ranade RG, Kanetkar SR: Extragenital heterologous malignant mixed müllerian tumor of primary peritoneal origin. Indian J of Pathol and Microbiol 2009;52:88-90.

3 Cokelaere K, Michielsen P, De Vos R, et al: Primary mesenteric malignant mixed mesodermal (Müllerian) tumor with neuroendocrine differentiation. Mod Pathol 2001;14:515-520.

4 Shah IA, Jayram L, Gani OS, et al: Papillary serous carcinoma of the peritoneum in a man: a case report. Cancer 1998;82:860-866.

5 Shaco-Levy R, Sion-Vardy N, Piura B: Primary peritoneal malignant mixed müllerian tumor associated with colonic adenocarcinoma. Eur J Gynaecol Oncol 2005;26:509-510.

6 Ma CJ, Yang SF, Huang CC, et al: Malignant mixed müllerian tumor of primary mesenteric origin associated with a synchronous ovarian cancer: case report and literature review. Eur J Gynaecol Oncol 2008;29:289-293.

7 Mikami M, Kuwabara Y, Tanaka K, et al: Malignant mixed müllerian tumor of primary mesenteric origin. Int J Gynecol Cancer 2005;15:1249-1253.

8 Garamvoelgyi E, Guillou L, Gebhard S, et al: Primary malignant mixed Müllerian tumor (metaplastic carcinoma) of the female peritoneum. A clinical, pathologic, and immunohistochemical study of three cases and a review of the literature. Cancer 1994;74:854-863.

9 Ober WB, Black MB: Neoplasm of subcoelomic mesenchyme: Report of two cases. AMA Arch Pathol 1955;59:698-705.

10 Wong L, Seen HT, Khootan HS, et al: Combined adjuvant cisplatin and ifosfamide chemotherapy and radiotherapy for malignant mixed müllerian tumors of the uterus. Int J Gynecol Cancer 2006;16:1364-1369. 